\title{
Imaging Cutaneous T-Cell Lymphoma with Optical Coherence Tomography
}

\author{
Hans Christian Ring Inger Merete Hansen Stamp \\ Gregor B.E. Jemec \\ Department of Dermatology, Roskilde Hospital, Health Sciences Faculty, \\ University of Copenhagen, Copenhagen, Denmark
}

\section{Key Words}

Lymphoma - Optical coherence tomography · Diagnostic imaging

\begin{abstract}
Aim: To investigate the presentation of a patch-stage cutaneous T-cell lymphoma (CTCL) using optical coherence tomography (OCT).

Methods: A patient with a patch caused by CTCL was photographed digitally, OCT-scanned and biopsied. A normal skin area adjacent to the patch was OCT-scanned for comparison, but not biopsied. The OCT image and the histological image were compared.

Results: The OCT images illustrated a thickened and hyperreflective stratum corneum. OCT also demonstrated several elongated hyporeflective structures in the dermis. The largest structure was measured to have a width of $0.13 \mathrm{~mm}$. A good immediate correlation was found between histology and OCT imaging of the sample.

Conclusion: The aetiology of the elongated structures is thought to be lymphomatous infiltrates. Similar findings have been described in ocular lymphoma and may therefore be an important characteristic of cutaneous lymphoma. It may further be speculated that the differences in OCT images may reflect the biological behaviour of the infiltrate. This observation therefore suggests that OCT imaging may be a relevant tool for the in vivo investigation of mycosis fungoides and other CTCLs, but in order to verify these observed patterns in OCT imaging, further investigations will be required.
\end{abstract}

\section{Introduction}

Optical coherence tomography (OCT) is a non-invasive imaging technology which produces micrometre-resolution images. The OCT technique is based on infrared light and interferometry to provide a high-resolution real-time/still cross-sectional image of different biological tissues. When investigating human skin, a classic layered 
recognisable and reproducible architecture is displayed to a depth of approximately $2 \mathrm{~mm}$ [1]. OCT was originally developed for ophthalmological purposes and was initially applied for eye length measurements. The first in vivo medical image was published by Fercher et al. [2] in 1993. Since then, the clinical application of OCT has rapidly gained ground in several other medical fields and has become routine in ophthalmology. In this case report, we aim to describe the OCT images of a patch-stage cutaneous T-cell lymphoma (CTCL) in order to explore the potential of the method.

\section{Methods}

A commercially available OCT system was used (VivoSight, Michelson Diagnostics, UK). This is a multi-beam system with a lateral resolution of $<7.5 \mu \mathrm{m}$, and a vertical resolution of $<10 \mu \mathrm{m}$. A CTCL patch was identified on the right side of the upper trunk. After obtaining informed consent the patch and an adjacent normal skin area were digitally photographed and OCT-scanned. The circle in fig. 1 a indicates where the OCT probe was located in relation to the patch. A subsequent biopsy of diseased tissue was performed.

\section{Case Presentation}

An 83-year-old woman was referred to the Department of Dermatology of Roskilde Hospital on suspicion of CTCL. She presented several asymptomatic erythematous, slightly pruritic patches, asymmetrically scattered on the trunk. A biopsy verified the suspicion of mycosis fungoides. The investigated patch measured $7 \times 3 \mathrm{~cm}$.

Compared to an adjacent non-affected skin area, OCT displayed a hyperreflective and thickened stratum corneum. The width of the affected and non-affected stratum corneum was measured to 0.027 and $0.011 \mathrm{~mm}$, respectively. The OCT image demonstrated a remarkable hyporeflective oblong structure in the papillary dermis and several other more blurred dark structures situated more deeply in the dermis. The largest of these structures was measured to have a width of $0.13 \mathrm{~mm}$.

The lesional biopsy revealed well-differentiated epithelium with a slight hyperkeratinisation. The epidermis and dermis showed a massive infiltrate of lymphocytic cells predominantly located in a superficial subepidermal band embedded in fragmented collagenous connective tissue. The fragmented collagenous connective tissue extended through the depth of the biopsy.

\section{Discussion}

Histology still represents the gold standard for micromorphic investigation, but skin biopsy causes trauma, even if minimal, and pain and may also have limitations such as the inability to repeat investigations at the exact same sites. OCT is a non-invasive high-resolution imaging method that is routinely used in ophthalmology where it has provided important insight into the functional anatomy of the eye, and is currently being explored as a diagnostic tool in dermatology.

Several authors have described the OCT features of various dermatological skin diseases, and for tumours such as basal cell carcinoma and actinic keratosis considerable progress has been made. In OCT imaging, basal cell carcinomas are found to display distinctive rounded hyporeflective structures often demarcated by a circular hyperreflective fringe. OCT images of actinic keratosis show a disarray of the epidermis with white dots and streaks corresponding to hyperkeratosis [3]. 
Mycosis fungoides is an uncommon CTCL characterized cytomorphologically by the proliferation of small- to medium-sized pleomorphic lymphocytes infiltrating the skin in a subepidermal band. Intraepidermal clustering of lymphocytes ('Pautrier's microabscesses') is considered the hallmark of the disease though it may be absent from more advanced lesions [4]. Clinically, mycosis fungoides is a differential diagnosis to a number of inflammatory dermatoses such as dermatitis or psoriasis. To the best of the authors' knowledge, the potential of OCT to identify distinctive features of CTCL has never been investigated.

We studied a patch-stage mycosis fungoides on the right side of the upper trunk. The OCT images illustrated a thickened and hyperreflective stratum corneum. This may reflect the keratinisation of the patch. Hyperkeratosis is also seen in psoriasis and is characterised in OCT by a more intense backscattering from the surface layers [5]. OCT also demonstrated several elongated hyporeflective structures in the dermis. It was not possible to delimit this fragmented pattern as it exceeded the penetration depth of OCT imaging of $2 \mathrm{~mm}$. A good immediate correlation was found between histology and OCT imaging of the sample (fig. 1).

Similar OCT characteristics have been described in ocular lymphoma. Recently, an OCT study of intraocular lymphoma illustrated similar features in the form of subretinal spaces, corresponding to a dense lymphocytic infiltrate [6]. These elongated areas may therefore be an important characteristic of cutaneous lymphoma. They appear similar to the spaces seen in the histological slides, although the latter are most likely artefacts which occur during processing of the tissue for histology. Recently, Banzhaf and Jemec [7] showed that more rounded dermal hypoechoic areas may be found in popular or diffuse granuloma annulare, and it may be speculated that the differences in OCT images may reflect the biological behaviour of the infiltrate.

This observation therefore suggests that OCT imaging may be a relevant tool for the in vivo investigation of mycosis fungoides and other CTCLs, but in order to verify these observed patterns in OCT imaging, further investigations will be required. 


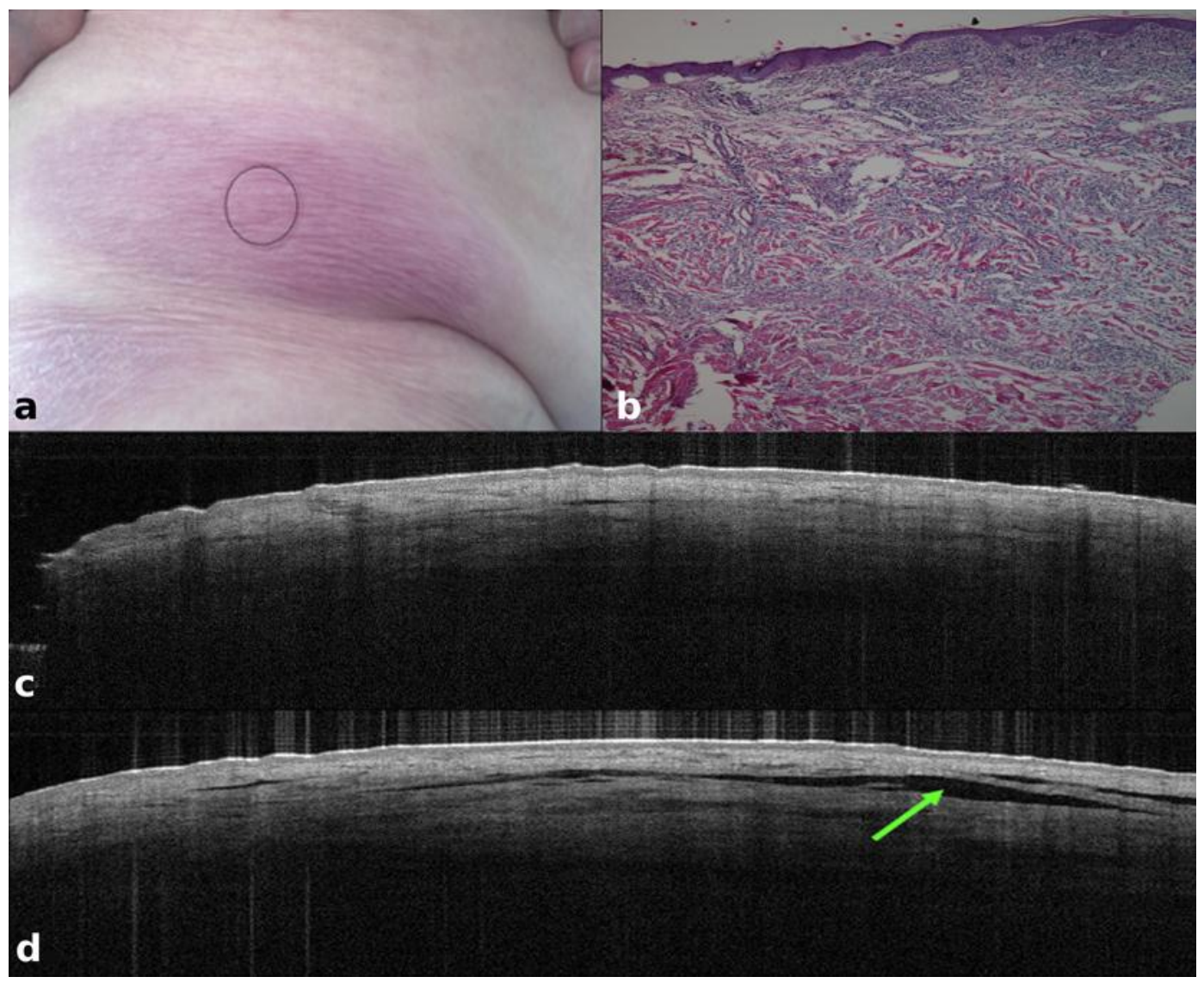

Fig. 1. a An erythematous patch identified on the right side of the upper trunk. The circle indicates exactly where the OCT probe was located in relation to the patch. $\mathbf{b}$ Hematoxylin-eosin stain of biopsy showing a massive lymphocytic infiltrate predominantly located in a superficial subepidermal band embedded in a fragmented connective tissue. c An OCT image of a normal skin area adjacent to the patch displaying the classic layered architecture of the skin without any pathological features. $\mathbf{d}$ The OCT image of the CTCL patch reveals a thickened and hyperreflective stratum corneum. Note the hyporeflective oblong structure in the papillary dermis and several other more blurred dark structures situated more deeply in the dermis. 


\section{References}

$\checkmark 1$ Fujimoto JG: Optical coherence tomography for ultrahigh resolution in vivo imaging. Nat Biotechnol 2003;21:1361-1367.

-2 Fercher AF, Hitzenberger CK, Drexler W, et al: In vivo optical coherence tomography. Am J Ophthalmol 1993;116:113-114.

-3 Mogensen M, Nurnberg BM, Forman JL, et al: In vivo thickness measurement of basal cell carcinoma and actinic keratosis with optical coherence tomography and 20-MHz ultrasound. Br J Dermatol 2009;160:1026-1033.

4 Cerroni L, Gatter K, Kerl H: An Illustrated Guide to Skin Lymphoma. Blackwell Publishing, 2004.

5 Welzel J, Bruhns M, Wolff HH: Optical coherence tomography in contact dermatitis and psoriasis. Arch Dermatol Res 2003;295:50-55.

6 Liu TY, Ibrahim M, Bittencourt M, et al: Retinal optical coherence tomography manifestations of intraocular lymphoma. J Ophthalmic Inflamm Infect 2012, Epub ahead of print.

7 Banzhaf C, Jemec GB: Imaging granulomatous lesions with optical coherence tomography. Case Rep Dermatol 2012;4:14-18. 


\section{Erratum}

In the article by Ring HC, Hansen Stamp IM, Jemec GB: Imaging Cutaneous T-Cell Lymphoma with Optical Coherence Tomography. Case Rep Dermatol 2012;4:139-143 (DOI: 10.1159/000340010), the following disclosure statement needs to be added:

Michelson Diagnostics provided the OCT system and technical support for this study. 